\title{
Functional enrichment of gut microbiome by early supplementation of Bacillus based probiotic in cage free hens: a field study
}

\author{
Samiullah Khan and Kapil K. Chousalkar *i)
}

\begin{abstract}
Background: The chicken gut microbiota passes through different stages of maturation; therefore, strengthening it with well characterised probiotics increases its resilience required for optimum gut health and wellbeing. However, there is limited information on the interaction of Bacillus based probiotics with gut microbial community members in cage free laying chickens both in rearing and production phases of life. In the current study, we investigated the changes in the gut microbiome of free range hens in the field after Bacillus based probiotic supplementation.

Results: Overall, at phylum level, probiotic supplementation increased the populations of Bacteroidetes and Proteobacteria mainly at the expense of Firmicutes. The population of Bacteroidetes significantly increased during the production as compared to the rearing phase, and its higher population in the probiotic-supplemented chickens reflects the positive role of Bacillus based probiotic in gut health. Core differences in the beta diversity suggest that probiotic supplementation decreased microbial compositionality. The non-significant difference in alpha diversity between the probiotic and control chickens showed that the composition of community structure did not change. No Salmonella spp. were isolated from the probiotic supplemented birds. Egg internal quality was significantly higher, while egg production and body weight did not differ. Functional prediction data showed that probiotic supplementation enriched metabolic pathways, such as vitamin B6 metabolism, phenylpropanoid biosynthesis, monobactam biosynthesis, RNA degradation, retinol metabolism, pantothenate and CoA biosynthesis, phosphonate and phosphinate metabolism, AMPK signaling pathway, cationic antimicrobial peptide (CAMP) resistance and tyrosine metabolism.
\end{abstract}

Conclusions: Overall, age was the main factor affecting the composition and diversity of gut microbiota, where probiotic supplementation improved the abundance of many useful candidates in the gut microbial communities. The generated baseline data in the current study highlights the importance of the continuous use of Bacillus based probiotic for optimum gut health and production.

Keywords: Gut microbiome, Free-range chicken, Probiotic, Microbial diversity, Microbial abundance

\section{Background}

In a healthy state, the assemblage of viruses, bacteria, archaea, and fungi forms a host microbiome that can mediate biological functions ranging from energy metabolism to immune response biomolecules production [1-4]. A stable consortium of commensal gut microbial

\footnotetext{
* Correspondence: Kapil.chousalkar@adelaide.edu.au

School of Animal and Veterinary Sciences, The University of Adelaide, Roseworthy, South Australia 5371, Australia
}

communities is associated with pathogen exclusion [5], as reduced diversity has been shown to increase the risk of infection [6, 7]. The $16 \mathrm{~S}$ rRNA metagenomics data have disclosed differences in community membership of different age groups, differences that contribute to pathophysiological functions in a host. For example, metagenomics data from murine, human and chicken models indicate that the host-microbiota cross-talk influences response to cell injury [8], affects energy balance [9], supports the

(c) The Author(s). 2021 Open Access This article is licensed under a Creative Commons Attribution 4.0 International License, which permits use, sharing, adaptation, distribution and reproduction in any medium or format, as long as you give appropriate credit to the original author(s) and the source, provide a link to the Creative Commons licence, and indicate if changes were made. The images or other third party material in this article are included in the article's Creative Commons licence, unless indicated otherwise in a credit line to the material. If material is not included in the article's Creative Commons licence and your intended use is not permitted by statutory regulation or exceeds the permitted use, you will need to obtain permission directly from the copyright holder. To view a copy of this licence, visit http://creativecommons.org/licenses/by/4.0/. 
synthesis and biotransformation of isoprenoids, vitamins, xenobiotics, amino acids and glycans [10], provides colonisation resistance against pathogens $[6,11]$ and influences the maturation of immune system [12]. In the host, the fermentation process is mainly achieved by microbiota through the production of gut metabolites that include indole and its derivatives, linoleic acids, tryptamine, shortchain fatty acids (SCFAs) and vitamins [13, 14].

In layer chickens, gut microbiota influences performance and resistance to pathogens, such as Salmonella and Campylobacter. For example, an increased abundance of Faecalibacterium resulted in clearing Salmonella Typhimurium from the gut [6]. Higher abundance levels of Fusobacteria and Bacteroides were associated with increased egg production [15]. Gut health is maintained partly by the resident gut microbiota that provides the first line of resistance against pathogen colonisation. However, the composition and diversity of gut microbiota vary with genotype [16], rearing conditions, age $[17,18]$ and stress factors [19-21]. Affected by body weight, certain species of Lactobacillus, and Lactococcus lactis and Bacillus thermoamylovorans were significantly higher in abundance in low body-weight laying hens, suggesting the role of these bacteria in body composition of the host [22]. Assessing the age effect on gut microbiota composition, compared to 8 weeks of chickens' age, Bacteroidetes and Firmicutes were more abundant in the gut of 30 week laying hens [17]. A comparative analysis of the gut microbiota of cage and free-range production systems showed a higher abundance of Bacteroidetes in free-range laying hens [23]. This suggests that a range of factors affect gut microbiota; therefore, strategies that help in enhancing gut health will result in lower colonisation of the gut by pathogens and improve hens' performance.

One way to improve the diversity and composition of gut microbial communities is to supplement the diet with probiotics [13]. Probiotics are viable bacteria that maintain gut health through the production of organic acids [24], prime the immune system [25, 26] and help in the saturation of enterocytes [27] for pathogen colonisation resistance. Bacteria such as Lactobacillus, Bacillus, Bifidobacterium and Streptococcus are used for probiotics formulation that are commercially available for poultry industry. Currently, there are limited reports on the development and maturation of gut microbiota in cage free/free-range laying chickens in the field [17, 28, 29]. However, the microbiota of free range production is more diverse than cage chickens [17]. Finding microbial communities associated with the layer performance, and whether the Bacillus based probiotic plays a role in improving the diversity and composition of gut microbiota in free-range laying chickens will help in devising strategies for improving gut health for product safety. The optimised probiotic can be used for the control of food safety pathogens, such as Listeria, Clostridium, Salmonella and Campylobacter. Therefore, we hypothesised that if used from the day of a hatch in the field, Bacillus based probiotic will improve the composition of gut microbiota, enhance egg quality and reduce gut pathogen colonisation in cage free hens in the field. The chosen Bacillus based probiotic is commercially available as a premix for poultry feed. The main objective of this study was to test the effects of Bacillus based probiotic on gut health and layer performance in free-range production system.

\section{Methods}

\section{Animal ethics and experimental design}

The experimental work was approved by the Animal Ethics Committee at The University of Adelaide under approval number S-2019-109. Faecal swab collections were performed as per standard operative procedures approved by the Animal Ethics Committee.

A commercial farm was selected based on the willingness of the farm manager and the farm set up that was appropriate for this study. Prior to placing the chicks, environmental swabs $(n=40)$ were collected from empty rearing sheds (labelled as control and probiotic supplemented) before and after the clean-up procedures to determine the contamination level of Salmonella spp. (if any). The experimental flock (Hyline Brown) was distributed into 2 rearing sheds on a pullet-rearing farm from day 1 of placement with 10,860 and 10,457 chicks in the control and probiotic supplemented sheds, respectively. The stocking density was $30 \mathrm{birds} / \mathrm{m}^{2}$. One shed acted as a control, while chicks in the other shed received a premix of Bacillus based probiotic at a rate of $1 \mathrm{~g} / \mathrm{kg}$ of feed from day 1 until the termination of the experiment (week 36 of flock age). The commercially available probiotic was composed of Bacillus subtilis DSM 32324, Bacillus subtilis DSM 32325 and Bacillus amyloliquifaciens DSM 25840. This probiotic was chosen as its continuous supplementation in a pen trial resulted in the overall lower shedding level of Salmonella Typhimurium [6]. Birds in both the sheds received vaccines against coccidiosis, infectious bronchitis, avian encephalomyelitis, New Castle disease, egg drop syndrome, infectious laryngotracheitis and fowl cholera. The pullets were raised on a concrete floor and they received chick starter feed from 1 to 6 weeks, a grower from 7 to 12 weeks, a developer from 13 to 14 weeks, pre-lay from 15 to 17 weeks and peak lay diet from week 18 onwards (Additional file 1; Tables S1-5). Prior to shifting pullets to a production farm, the production sheds were swabbed $(n=40)$ to determine the contamination level of Salmonella spp. (if any). The pullets were shifted to free-range production sheds (control and probiotic supplemented) 
at 16-week of flock age. The distance between the rearing and production farms is approximately $30 \mathrm{~km}$. Fresh faeces $(n=20)$ for DNA extraction, faecal swabs $(n=20)$ and environmental dust swabs $(n=10)$ for Salmonella isolation from each treatment group at each sampling time point were collected at day 1 (meconium samples), 5, 21, week 6, 12, 16 (Day 1 and 5 after shifting), 18, 24, 30 and 36 of flock age. Therefore, for 11 sampling timepoints, a total of 440 faecal DNA samples, 440 faecal swab samples and 220 environmental dust swab samples were processed during this study. Once in lay, eggs $(n=$ 30) from each treatment group were collected at week 24, 30 and 36 of flock age and processed for egg quality measurements. Faecal swab samples were collected in 4 $\mathrm{mL}$ buffered peptone water (BPW, ThermoFisher Scientific, Australia), while environmental swabs (Whirl-Pak "Speci-Sponge, ThermoFisher Scientific, Australia) were soaked in $20 \mathrm{~mL}$ BPW and individual swabs were dragged to cover at least $1 \mathrm{~m}^{2}$ area in the shed including exhaust fans and covering boards of nest boxes. Shoe covers from each shed were soaked in $150 \mathrm{~mL} \mathrm{BPW}$ and processed for Salmonella isolation.

\section{Processing of faecal and environmental swabs for Salmonella isolation}

The collected samples were processed for the isolation of Salmonella spp. as outlined in Additional file 1. Briefly, the faecal and environmental dust swab samples were incubated overnight and then enriched in Rappaport-Vassiliadis soya peptone (RVS) broth for the selective growth of Salmonella. The RVS overnight incubated samples were streaked on brilliance Salmonella and xylose lysine deoxycholate agar for the "YES" or "NO" confirmation of Salmonella. A miniaturized most probable number (mMPN) method was used to semiquantify the load of Salmonella from the confirmed positive samples. Confirmed Salmonella isolates were further processed for traditional PCR using an invasion (invA) and TSR3 genes specific primers.

\section{Faecal DNA extraction and 16S rRNA sequencing}

Total DNA from the collected faeces was extracted following a modified protocol of QIAamp FAST DNA Mini Kit (Additional file 1). The undiluted DNA samples $(n=$ 440; 20 samples per treatment group at each sampling for a total of 11 time-points) were sequenced by the Ramaciotti Centre for Genomics (University of New South Wales, Australia) for $16 S$ rRNA metagenome sequencing and subsequent data analysis for the generation of operational taxonomic units (OTUs) table. For microbial profiling, the hypervariable region (V3-V4) of the $16 S$ rRNA gene was sequenced using a barcoded primer pair (341F: 5'-CCTACGGGNGGCWGCAG-3'; 805R: 5'-GACTACHVGGGTATCTAATCC-3').
16S rRNA library preparation and Illumina sequencing For individual faecal DNA samples, the $16 S \mathrm{rRNA}$ library was prepared by a barcoding PCR in a $25 \mu \mathrm{L}$ final reaction volume that contained $12.5 \mu \mathrm{L}$ of KAPA HiFi HotStart Readymix PCR buffer (Kapa Biosystems), $9.5 \mu \mathrm{L}$ of PCR grade water, $1 \mu \mathrm{L}$ of each of the barcoded forward and reverse primers and $1 \mu \mathrm{L}$ of faecal DNA template. The cycling conditions in SimpliAmp Thermal Cycler (Applied Biosystems) were: initial denaturation at $95^{\circ} \mathrm{C}$ for $3 \mathrm{~min}$, denaturation at $95^{\circ} \mathrm{C}$ for $30 \mathrm{~s}$, annealing at $55^{\circ} \mathrm{C}$ for $30 \mathrm{~s}$ and elongation at $72^{\circ} \mathrm{C}$ for $30 \mathrm{~s}$ for a total of 35 cycles. A final elongation at $72^{\circ} \mathrm{C}$ for 5 min was included at the end of the cycles. The PCR amplicons were normalised and pooled using the SequalPrep Normalization Plate Kit (ThermoFisher Scientific, Australia) as per the manufacturer's guidelines. The PCR library was purified using AxyPrep Mag PCR Clean-Up Kit (Fisher Biotec, Australia) as per the manufacturer's protocol. The quality and concentration of the pooled library were assessed by Qubit, and the library size was estimated on an Agilent 2200 TapeStation (Integrated Science, Australia). From the pooled library, primerdimer was removed by the Agencourt AMPure XP Bead Clean-up kit. The pooled library was sequenced on Illumina MiSeq using the MiSeq Reagent Kit v3 with a $2 \times$ $300 \mathrm{bp}$ run format as per the manufacturer's protocol. For the MiSeq runs, custom primers were added to the reagent cartridge for Read1, Index and Read2.

\section{Microbial community data analysis and statistical tests}

Raw sequencing reads (Fastq files) were processed with the OTUreporter v1.0.1-beta (5576d57) pipeline base on mothur (v1.39.5) [30, 31]. Briefly, the reads were quality filtered and assigned to their respective samples. Sequences were trimmed according to the MiSeq SOP [31] and only those with a length between 100 and $473 \mathrm{bp}$ were retained, while longer than $8 \mathrm{bp}$ homopolymer containing sequences were removed. For chimera removal, a chimera.vsearch script in mothur was used [32]. The sequences were aligned and classified against the SILVA reference alignment (v132) [33] and non-bacterial lineages not targeted by the barcoded primer pair (i.e. unknown, mitochondria, archaea, chloroplast, mitochondria and eukaryote) were removed. Sequences were grouped into OTUs based on 97\% similarity using the OptiClust algorithm [34] and subsampled based on the sample with the lowest number of reads $(n=11,092)$. Sequencing error was assessed using the NO SEQ ERROR TEXT as a control in each run.

The OTU data were analysed in Calypso v 8.84 that functions based on $R$ packages vegan [35] for microbiota phylogenetic analysis of community composition, abundance and diversity by taking probiotic supplementation and flock age as independent variables. In the Calypso, the OTU data were normalised using cumulative-sum 
scaling (CSS) and expressed as $\log _{2}$ relative abundance to account for the non-normal distribution of taxonomic counts data. In the CSS normalisation, raw counts are divided by the cumulative sum of counts up to a percentile determined using a data-driven approach [36]. Genus/Phylum abundance was determined using oneway ANOVA. Alpha diversity was calculated using the Shannon index that takes into account the richness and evenness of microbial communities. Beta diversity was calculated using ANOSIM based on Bray-Curtis dissimilarity matrices. Redundancy analysis $(\mathrm{RDA}+)$ was used for measuring variation in structural composition of the microbial community. For RDA+ analysis, both the control and probiotic supplemented groups were taken together without applying any corrections. To understand the effect of rearing phases and laying effects, data were also analysed for diversity analysis between the control and probiotic treatment groups based on rearing (Day 1 to Week 12 samples) and production (Week 16 to Week 36 samples) phases, pre-lay (Week 16 and Week 18 samples) and early-lay (Week 24 and Week 30 samples) periods.

\section{Functional predictions of metabolic pathways through $16 S$ rRNA data}

The workflow of Tax4Fun2 [37] was used to predict the metabolic pathways of faecal microbiota. Differentially abundant features were identified using Welch's t-test inbuilt in the statistical analysis of taxonomic and functional profile (STAMP) software [38], where features were filtered using $q$ value $>0.05$, leaving only the significant features to be visualised. In STAMP, BenjaminiHochberg FDR was used for multiple test correction.

\section{Egg quality measurements}

The collected eggs were processed for measuring egg weight, shell weight, shell thickness, albumen height, Haugh Unit and yolk colour following a previously described method [39]. Briefly, Technical Supplies and Services (TSS, UK) QCH albumen height gauge was used for albumen height measurement, while yolk colour was measured by DSM Yolk Colour fan (scale 1-16). Shell thickness was measured by Mitutoyo Dial Comparator Gauge Model 2109-10 (Kawasaki, Japan). Haugh Unit (HU) was measured from the egg weight and albumen height by using the following equation [40]:

$\mathrm{HU}=100 * \log _{10}\left(\mathrm{HT}-1.7 * \mathrm{EW}^{\wedge} 0.37+7.6\right)$; where HT is albumen height ( $\mathrm{mm}$ ) and EW is egg weight (g).

In addition, at the farm, egg production was recorded daily, while body weight of 100 birds from each shed was recorded weekly. Where appropriate, egg quality data were analysed in StatView v5.0.1.0 with one- or two- way ANOVA. Level of significance was established at protected least significant difference $(\mathrm{PLSD})<0.05$.
Flock performance data (body weight, lay rate, egg weight and FCR) were visualised in Excel Workbook.

\section{Results \\ Metagenome data quality assessment and overall gut microbiota landscape}

The sequence data assessment showed that out of 440 faecal DNA samples, 8 samples (1.81\%) generated less than 10,000 reads per sample after passing the QC and chimera removal, and were therefore discarded from the downstream analysis (Additional file 2). Out of the 8 discarded samples, 7 samples were from day 1 meconium, which usually contains very little microbiota. After subsampling, the OTU coverage was $\geq 97 \%$. The slope of the rarefaction curve indicated that the sequenced data covered most of the microbial communities associated with faeces (Additional file 3: Fig. S1 a, b). Therefore, the quality evaluation steps showed that the data were robust and suitable for faecal microbiota composition and diversity analyses. The OTU table (in mothur format) and the metadata file (CSV format) are included with the manuscript for reproducibility of this study (Additional files 4 and 5).

\section{Phylogenetic variation in faecal microbiome affected by probiotic supplementation and flock age}

Comprehensively, the gut microbiota of the control and probiotic supplemented flocks was composed of 15 known bacterial phyla out of which Bacteroidetes, Firmicutes and Proteobacteria were the most abundant (Additional file 6: Fig. S2). Overall, the abundance of Bacteroidetes, Firmicutes and Proteobacteria accounted for $44.45,25.43$ and $11.56 \%$, respectively. Known phyla of bacteria that accounted for less than $1 \%$ of the total population included Actinobacteria (0.94\%), Fusobacteria $(0.73 \%)$, Epsilonbacteraeota $(0.67 \%)$, Tenericutes (0.59\%), Deferribacteres $(0.25 \%)$, Lentisphaerae $(0.21 \%)$, Verrucomicrobia $(0.19 \%)$, Spirochaetes $(0.18 \%)$, Synergistetes $(0.16 \%)$, Patescibacteria $(0.16 \%)$, Elusimicrobia $(0.11 \%)$ and Kiritimatiellaeota $(0.02 \%)$. Interestingly, probiotic supplementation significantly $(P<0.0001)$ decreased the overall abundance levels (\%) of Firmicutes and Spirochaetes and increased $(\mathrm{P}<0.0001)$ Elusimicrobia (Fig. $1 \mathrm{a}, \mathrm{b}$ ). Although the percentage for Cyanobacteria was calculated while using the Calypso software, it is not considered as a part of the normal gut microbiota as they are photosynthetic and their presence in gut samples is assumed to be derived from ingested cyanobacterial cells or chloroplast.

The commercial layer life cycle is mainly comprised of rearing and production phases, where the rearing period is mainly up to 16 weeks of age and the chickens are kept on dirt or concrete floor. Assessing the effects of the probiotic supplementation in the rearing phase, the 


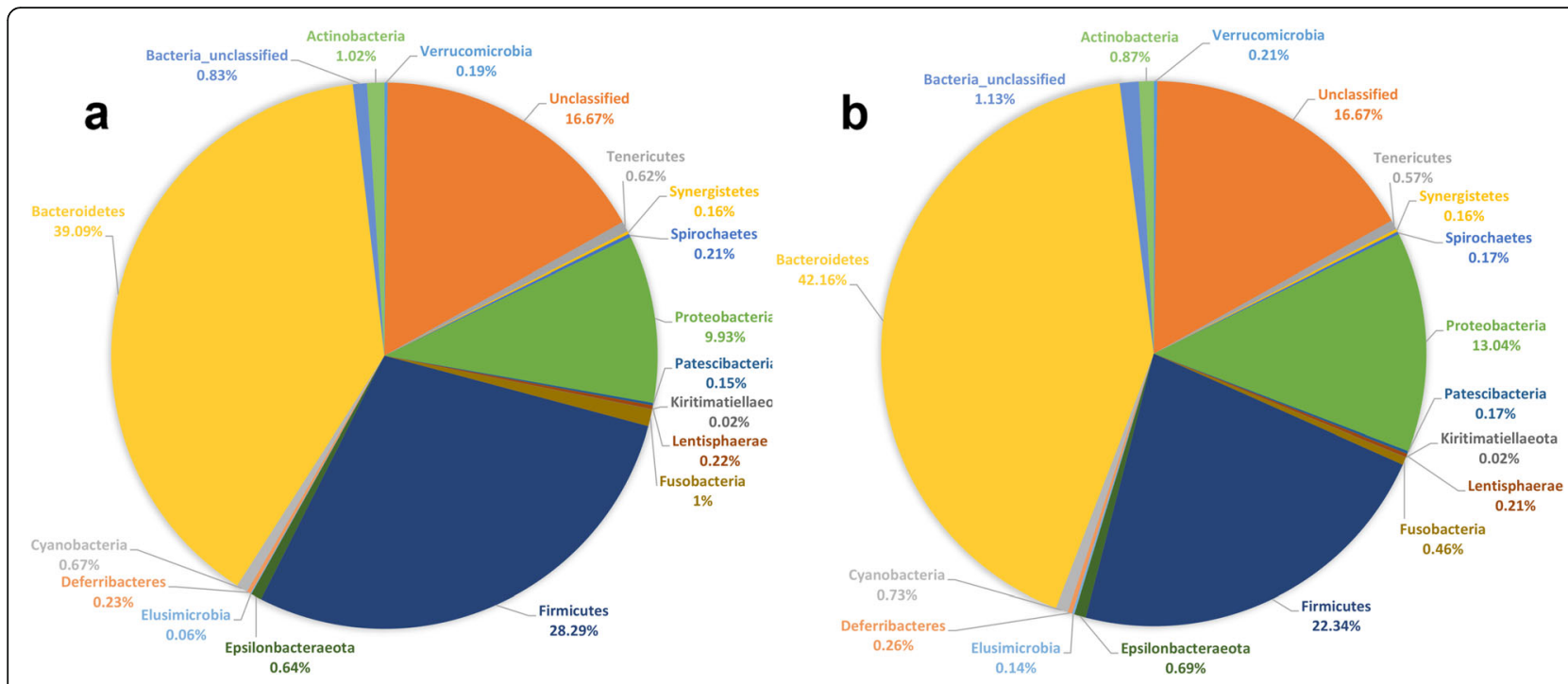

Fig. 1 Overall gut microbiota composition at the phylum level affected by probiotic supplementation. a). Microbial abundance (\%) in the control flock. b). Microbial abundance (\%) in the probiotic supplemented flock. For direct comparison between the control and probiotic supplemented groups, data obtained from the samples collected between day 1 and week 36 of flock age were analysed as a pool. For percent calculation of microbial abundance at phylum level, total sum scaling normalised but untransformed data obtained from Calypso software were visualised in Excel 2016 and the panel graphs were prepared in Graphpad prism v. 8.0.0

data showed that the abundance of Proteobacteria significantly increased while Firmicutes decreased (Fig. 2 a, b). In the production phase, in the probiotic supplemented flock, the abundance of Firmicutes remained lower, while Bacteroidetes increased compared with the control flock (Fig. 2 c, d).

Probiotic supplementation increased the abundance of numerous microbial communities at the genus level (Fig. 3). Overall, there was a significantly higher abundance of Elusimicrobium, Megasphaera, Parasutterella, Desulfovibrionaceae_unclassified, Paraprevotella, Succinatimonas, Bacteria_unclassified and Muribaculaceae_ge in the probiotic compared with the control flock (Fig. 3 a). Probiotic supplementation reduced the abundance levels of Sphaerochaeta and Rikenella. The effects of probiotic supplementation were more prominent in terms of affecting the abundance of multiple genera in the production than the rearing phase of life (Fig. $3 \mathrm{~b}, \mathrm{c}$ ). In the rearing phase, probiotic supplementation increased the abundance levels of Megasphaera, Parabacteroides, Parasutterella, Tannerellaceae_unclassified and Paraprevotella (Fig. 3 b), while in the production phase, in addition to these, probiotic supplementation increased the abundance levels of a range of microbial communities including Elusimicrobium, Butyricimonas, Bacteroides, Peptococcus and Faecalibacterium (Fig. 3 c). However, the abundance of Tannerellaceae_unclassified was not significantly altered in the production phase of life. In the production phase, probiotic supplementation resulted in the decreased abundance of microbial communities, such as Alistipes, Sphaerochaeta and Romboutsia.
The overall abundance of microbial communities (genus level) significantly different between the probiotic supplemented and control flocks were further investigated to understand the effects of probiotic in a longitudinal manner. The data showed that the probiotic supplementation affected the composition of gut microbiota with significant effects observed from Day 21 until Week 30 of flock age at least for one or more microbial communities (Fig. 4). The abundance levels of Elusimicrobium, Megasphaera, Parasutterella and Paraprevotella (Fig. 4 a-d), and Succinatimonas and Muribaculaceae_ge (Fig. 4 e, f) were significantly higher in the probiotic flock at the respective sampling periods.

Probiotic supplementation also significantly reduced the abundance levels of Sphaerochaeta and Rikenella (Fig. 4 g, h). The data showed that Sphaerochaeta mainly colonised the gut around week 16, while Rikenella started appearing from Day 21 of chickens' age. Overall, the age-wise analysis of the data showed that the colonisation time of different microbial communities varied with the nature of bacteria, where Bacteroides, Rikenellaceae_RC9_gut_group, Lactobacillus, Alistipes, EscherichiaShigella, Enterococcus, Clostridium sensu stricto and Enterococcaceae_unclassified were present in abundance in the gut from day one (Additional file 7: Fig. S3 and Additional file 8: Fig. S4). Interestingly, the abundance levels of Bacteroides, Rikenellaceae_RC9_gut_group, Lactobacillus and Alistipes (Additional file 7: Fig. S3 a d) in the gut of both in the control and the probiotic flocks remained consistent until week 36, while EscherichiaShigella, Enterococcus, Clostridium sensu stricto and 


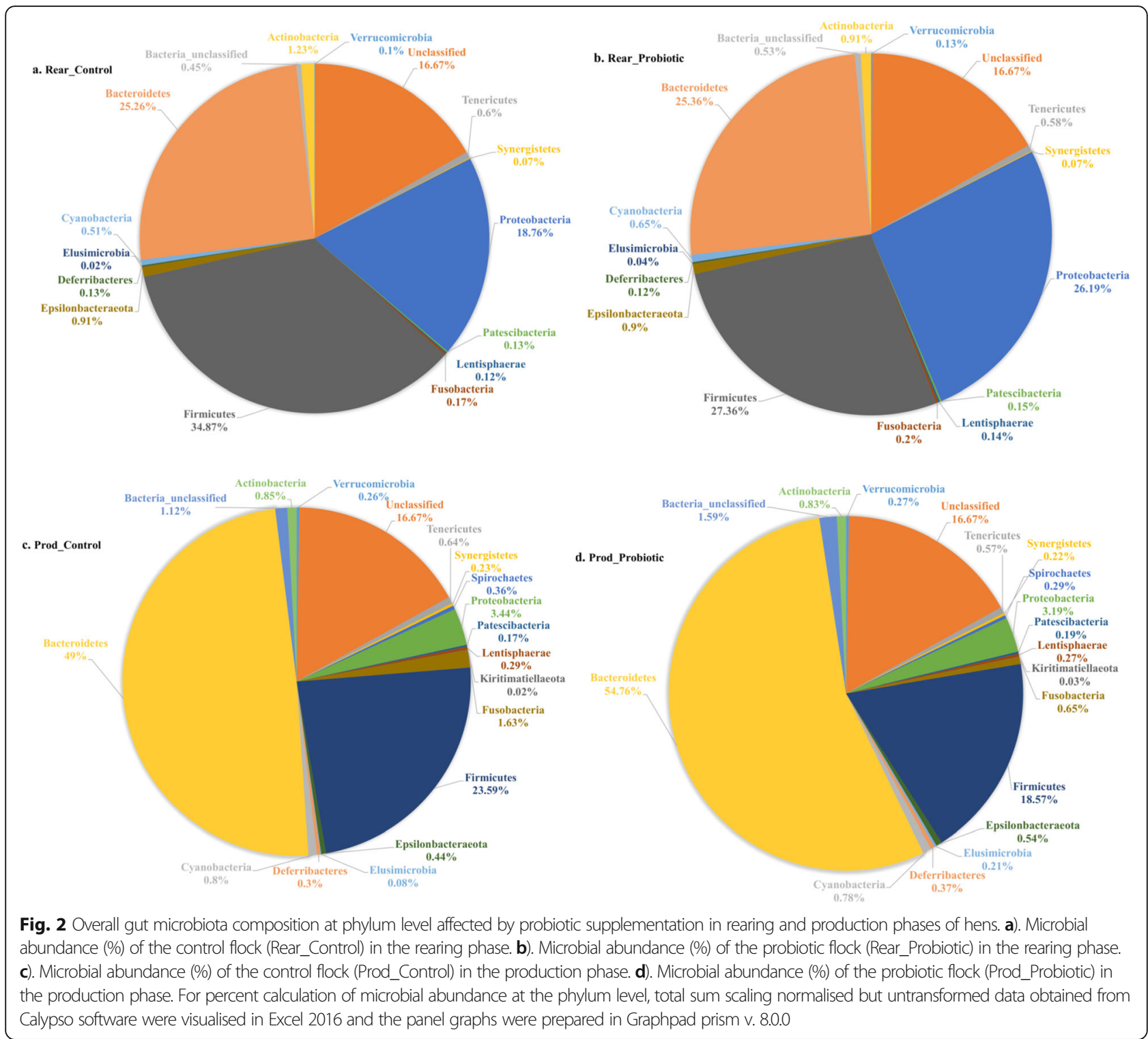

Enterococcaceae_unclassified decreased (Additional file 8: Fig. S4 a - d).

\section{Microbial community diversity affected by probiotic supplementation and flock age Microbial alpha diversity}

Shannon index is widely used to measure the alpha diversity of a community that includes both the number of present taxa (richness) and how evenly the taxa are distributed (evenness). Alpha diversity measures variation in the structure of microbial community within individual samples. Measured by the Shannon index and Richness, overall there was no significant variation $(P>0.05)$ in the community structure of individual samples collected from the probiotic and the control flocks (Fig. 5 a, b). However, taxa in the control group was significantly
$(P=0.039)$ evenly distributed (Fig. 5 c). The community structure was affected both by the probiotic supplementation and rearing conditions as the diversity, richness and evenness were significantly different within the samples collected from flocks in rearing and production phases (Fig. $5 \mathrm{~d}-\mathrm{f}$ ). Hormonal changes occurring due to sexual maturity and on-set of lay might affect the diversity of gut microbiota. To understand this effect, the samples collected at week 12 to week 30 of flock age were categorised into pre-lay and early-lay periods and analysed for alpha diversity of gut microbiota. The Shannon index values showed that laying phase did not significantly affect the overall alpha diversity of the taxa of each treatment group (Fig. 5 g). However, the richness of the taxa in the control group before lay showed significant variation, while after the onset of lay, the 


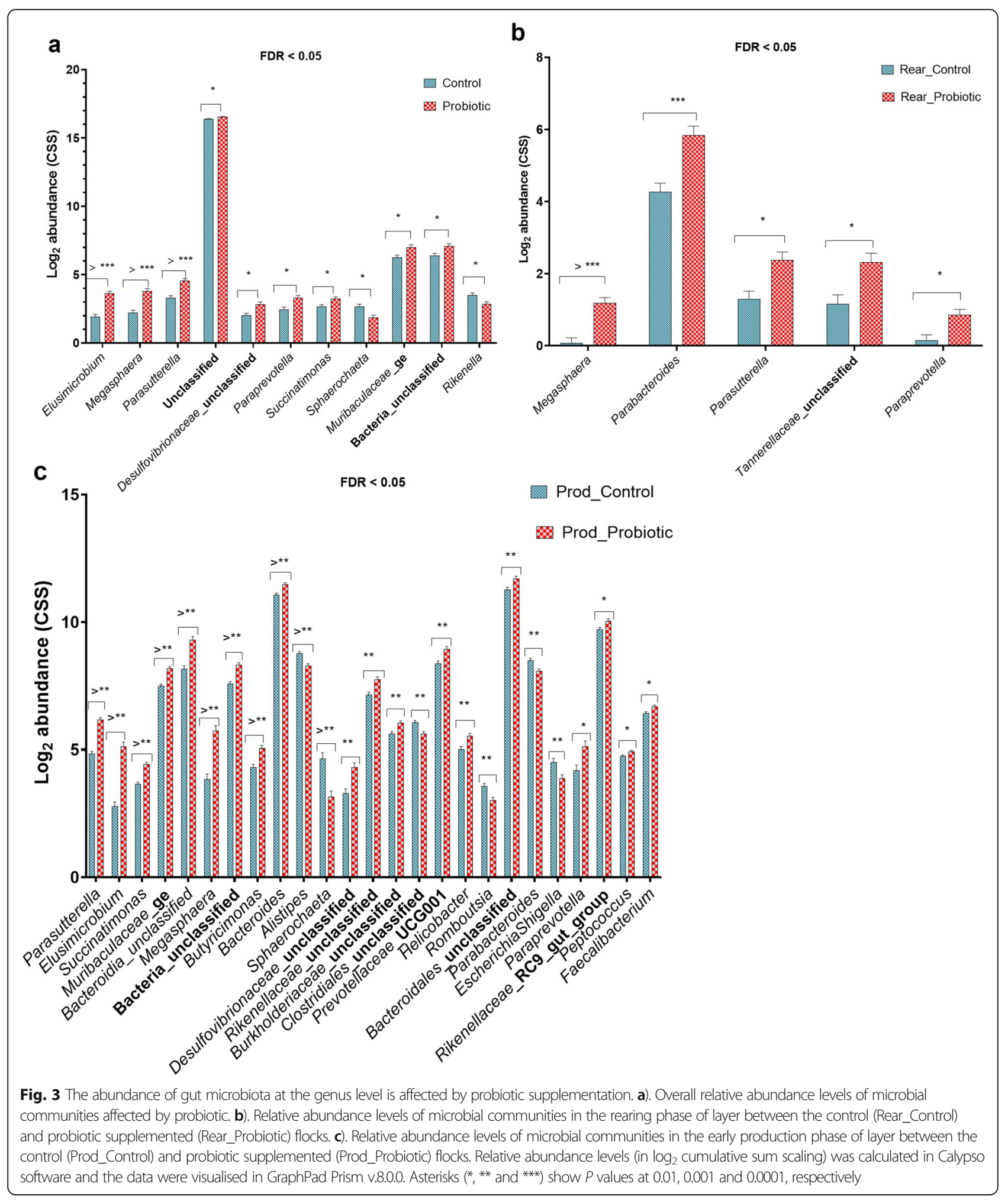

variation was significantly higher in the samples collected from the probiotic supplemented group (Fig. $5 \mathrm{~h}$ ). The evenness of the taxa was not significantly affected by the lay condition (Fig. 5 i).
The gut microbiota alpha diversity profile of the taxa of individual samples varied $\left(P<8.1 \mathrm{e}^{-126}\right)$ with flock age both in the probiotic supplemented and control flocks (Fig. 6). Both in the probiotic supplemented $(P=$ 

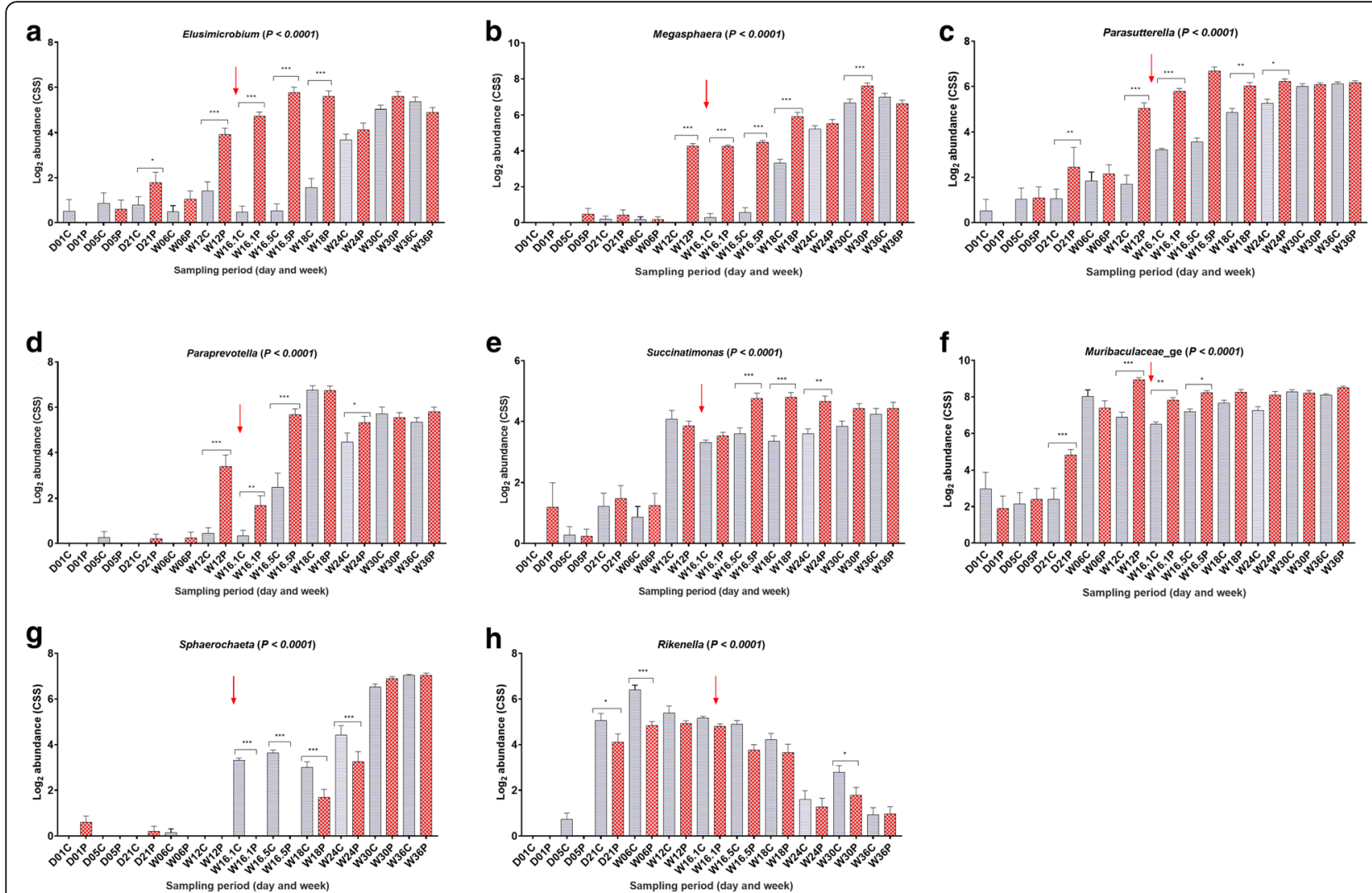

Fig. 4 Abundance of gut microbiota at genus level affected by probiotic supplementation at multiple sampling periods. a). Elusimicrobium. b). Megasphaera. c). Parasutterella. d). Paraprevotella. e). Succinatimonas. f). Sphaerochaeta. g). Muribaculaceae_ge. h). Rikenella. In each panel of Fig. 4, the letters " $\mathrm{D}$ " and " $\mathrm{W}$ " refer to day and week post-hatch, while the letters " $\mathrm{C}$ " and " $\mathrm{P}$ " refer to control and probiotic supplemented flocks, respectively. Asterisks $\left({ }^{*},{ }^{* *}\right.$ and ${ }^{* * *}$ ) show $P$ values at $0.01,0.001$ and 0.0001 , respectively

$\left.2.2 \mathrm{e}^{-99}\right)$ and control $\left(P=1.7 \mathrm{e}^{-78}\right)$ flocks, the alpha diversity of individual samples within each treatment group significantly varied from day 21 and onwards of flock age. Alpha diversity (measured by Shannon index) both in the control and probiotic supplemented groups increased from day 5 to week 16 before the flocks were moved to a free range farm for egg production. The probiotic supplemented flock showed a significantly lower alpha diversity on week 16.5 just after transportation to the production farm and on week 36 of flock age. This indicates that immediately after transportation, the alpha microbial diversity increased in the control group. For assessing the effects of feed on the alpha diversity of gut microbiota, the data were analysed on the basis of feed category phases, such as starter, grower, pre-lay and peak-lay. As expected, the overall alpha diversity (measured by Shannon index) showed significantly lower values $\left(P=7.2 \mathrm{e}^{-62}\right)$ in the starter phase of the feeding regimen (Additional file 9: Fig. S5 a). Richness (Additional file 9: Fig. S5 b) and evenness (Additional file 9: Fig. S5 c) also varied with feeding regimen and probiotic supplementation. As the birds were transported from rearing to the production farm, the samples obtained at week 12 and week 16 were assessed for the effects of stress on the alpha diversity of gut microbiota. Compared with the pre-transport, the post-transport individual samples diversity significantly varied $(P=0.0088)$ in the control flock (Additional file 9: Fig. S5 d). The richness of taxa at week 12 was significantly higher $(P=$ $4.6 \mathrm{e}^{-07}$ ) in the probiotic supplemented (Additional file 9: Fig. S5 e), while evenness was higher $\left(P=3.4 \mathrm{e}^{-06}\right)$ at week 16 in the control flock (Additional file 9: Fig. S5 f).

\section{Microbial beta diversity}

Beta diversity is used to measure similarity or dissimilarity of microbial communities between samples and; therefore, is a useful technique to capture changes in community composition based on the ecosystem. Measured by the ANOSIM Bray-Curtis dissimilarities matrix, the beta diversity of the flock that received the probiotic was lower $(P=0.002)$ than the control flock (Fig. 7 a). Compared with the control, beta diversity was significantly lower in the probiotic supplemented group for the samples collected from day 5 on-wards (Fig. 7 b). Both in the control and probiotic supplemented groups, lowest diversity was recorded in the samples collected 


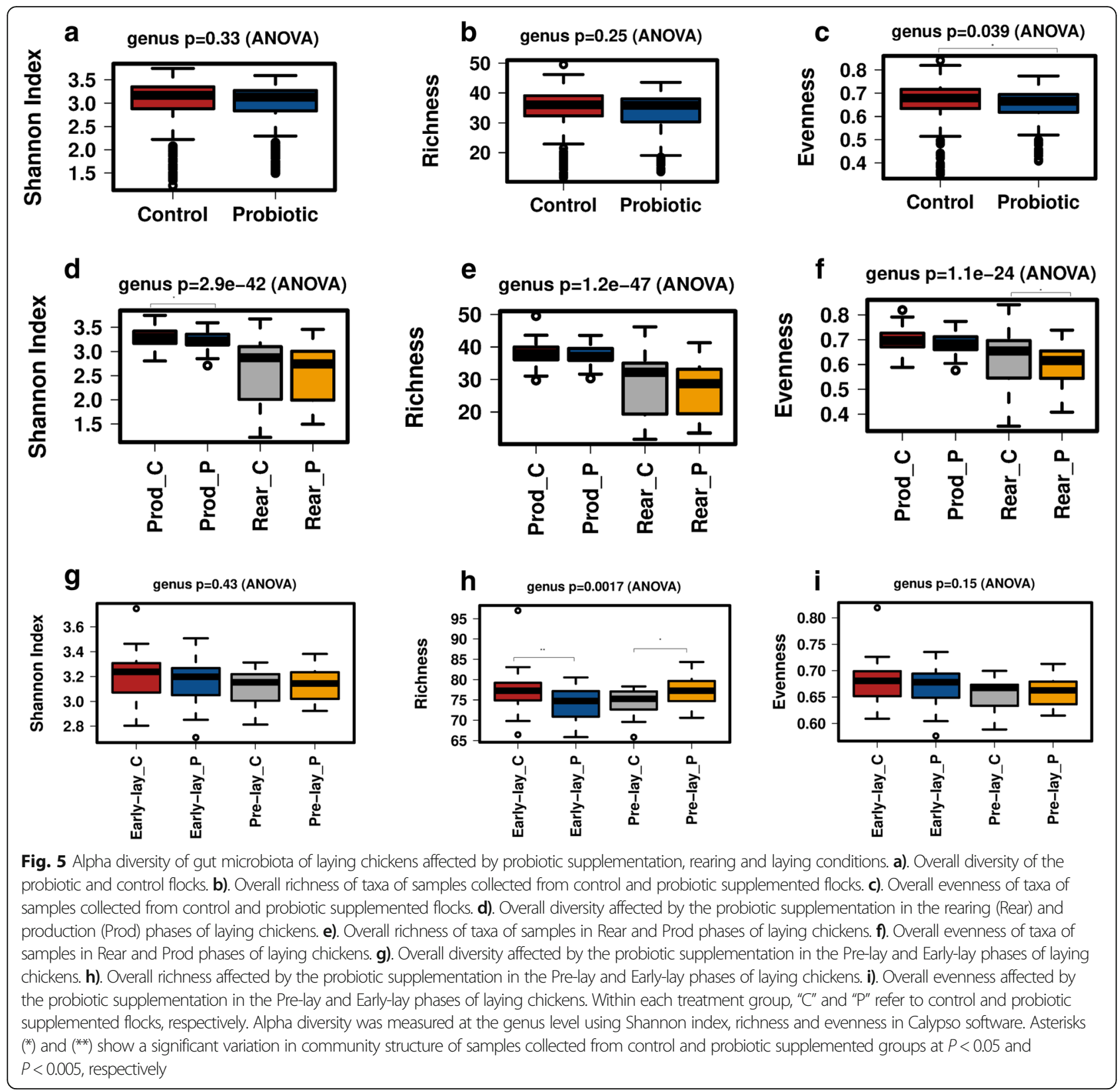

immediately post-transportation ( 16.1 week of flock age) to the production farm. Assessing the age effect on the beta diversity, the data showed that the community structure becomes less diverse with the flock age (Fig. 7 b). The beta diversity of the gut microbiota in the production was lower than the rearing phase, whereas the probiotic supplementation significantly reduced $(P=$ 0.001 ) the beta diversity in the production phase of the chickens (Fig. 7 c). Comparing the beta diversity of the gut microbiota in the pre-lay and early-lay phases showed that probiotic supplementation reduced $(P=$ 0.001 ) the beta diversity in both phases (Fig. $7 \mathrm{~d}$ ). The feeding regimen data showed that the beta diversity of the gut microbiota was lower $(P=0.001)$ when the flocks were on prelay and peak lay diets (Fig. 7 e). However, these data should be interpreted carefully, as we did not sample the flocks on exact time-points just before and after changing the diets. Therefore, age might have been a confounding factor here. Interestingly, when the data were assessed for understanding the transportation stress effect, the probiotic supplemented flock showed lower $(P=0.001)$ diversity post-transportation (Fig. $7 \mathrm{f}$ ).

To understand that how different was the microbial community structural composition between the probiotic and control groups collected at different timepoints, redundancy analysis (RDA+) was performed. The 


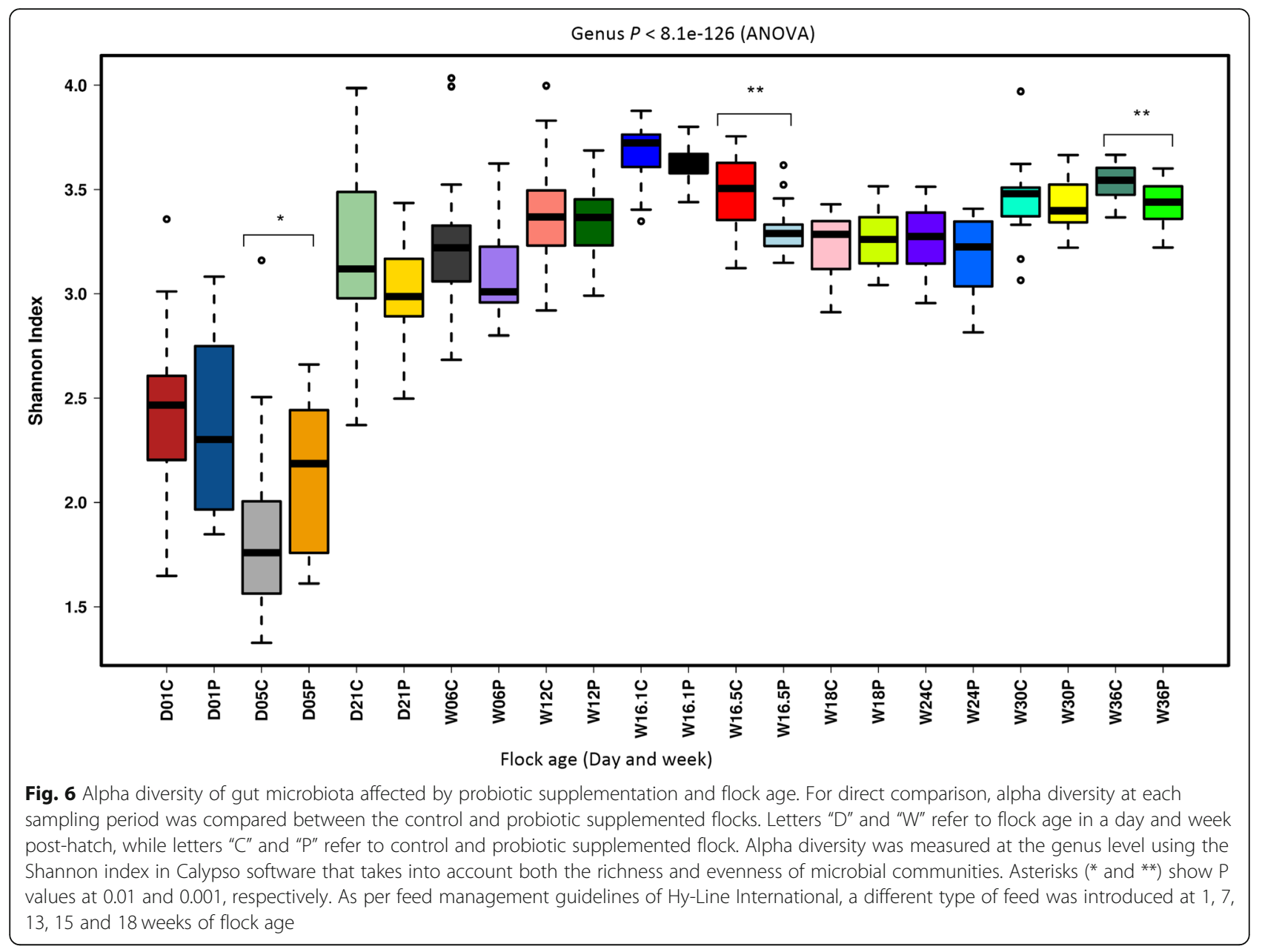

community structure of the probiotic and control flocks overlapped with each other but was significantly $(P=$ 0.005 ) different (Fig. 8 a). The community structure changed $(P=0.001)$ with flock age both in the probiotic supplemented and control flocks (Fig. 8 b), whereas rearing conditions had a profound effect $(P=0.001)$ on it (Fig. 8 c). The community structure changed $(P=0.001)$ in composition with a change in diet regimen (Fig. $8 \mathrm{~d}$ ).

\section{Functional prediction of gut microbial communities based on 165 rRNA data}

The effects of the probiotic on gut microbial metabolic pathways were assessed by performing functional predictions analysis in Tax4Fun2 using the KEGG orthologies (KOs) database and STAMP for Welch's t-test for calculating the level of significance between the treatment groups. The data were mapped to 345 functional metabolic pathways (Additional file 10). Functional metabolic pathways, such as vitamin B6 metabolism, lipopolysaccharide biosynthesis, phenylpropanoid biosynthesis, monobactam biosynthesis, RNA degradation, retinol metabolism, pantothenate and CoA biosynthesis, phosphonate and phosphinate metabolism, AMPK signaling pathway, cationic antimicrobial peptide (CAMP) resistance and tyrosine metabolism were significantly enriched in the probiotic supplemented compared with the control flock (Fig. 9).

The functional prediction of metabolic pathways data were also compared between the probiotic supplemented and control flocks at rearing and production phases of life. Less number of significantly enriched pathways in the probiotic treatment group in the rearing was observed than in the production phase of the layer chickens (Additional file 12: Fig. S7 and Additional file 13: Fig. S8). The common metabolic pathways significantly enriched in the probiotic supplemented flock both in the rearing and production phases included retinol metabolism, pantothenate and CoA biosynthesis, 2-oxocarboxylic acid metabolism and naphthalene degradation. Metabolic pathways, such as tropane, piperidine and pyridine alkaloid biosynthesis, nitrogen metabolism, tyrosine metabolism, lipopolysaccharide biosynthesis, methane metabolism, nonribosomal peptide structures and C5Branched dibasic acid metabolism were significantly enriched in the probiotic supplemented group in the rearing phase 


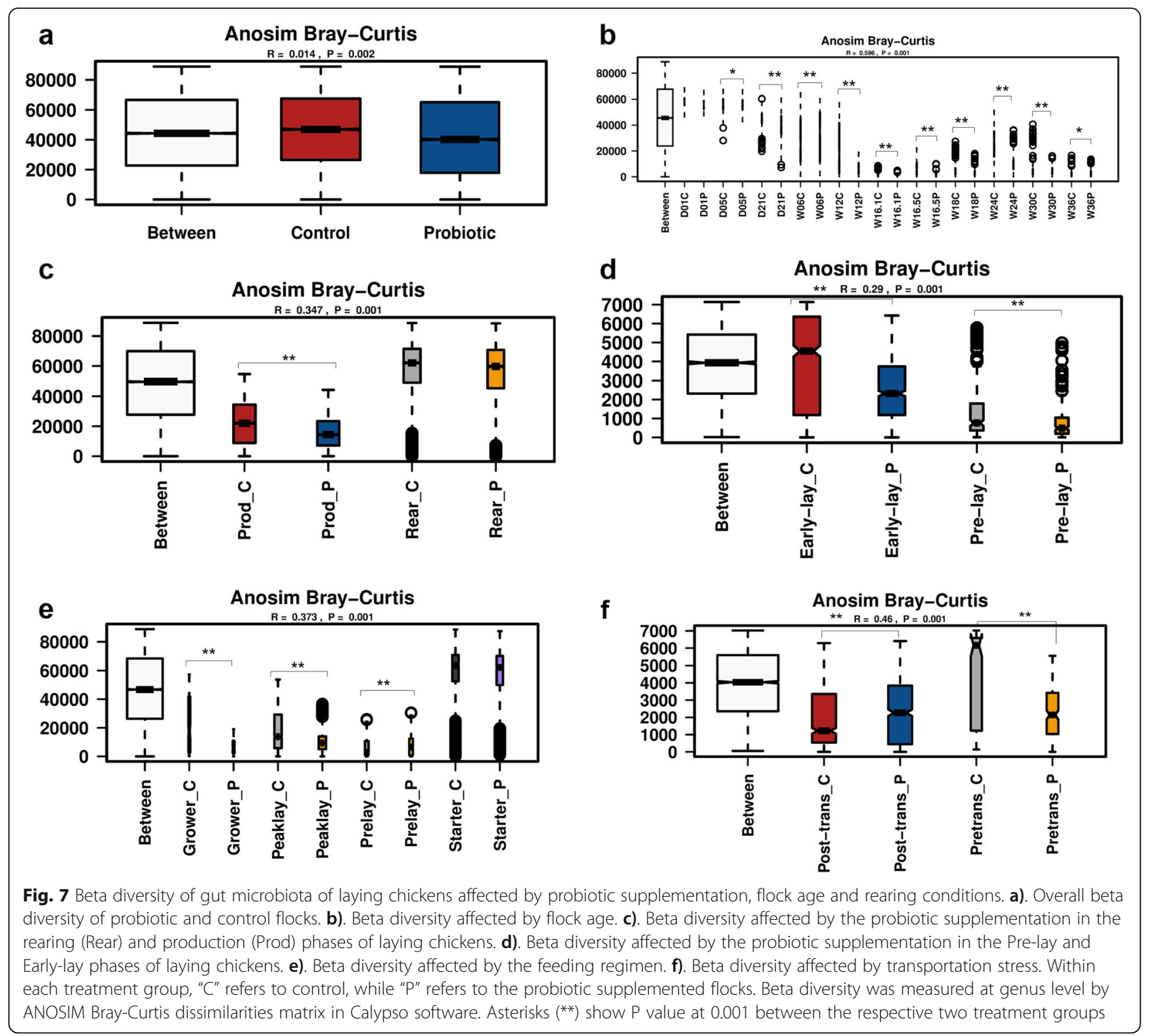

only (Additional file 12: Fig. S7). Metabolic pathways such as $\mathrm{N}$-glycan biosynthesis, plant-pathogen interaction, RNA degradation, vitamin B6 metabolism, AMPK signaling pathway, "phenylalanine, tyrosine and tryptophan biosynthesis", Dglutamine and D-glutamate metabolism, biosynthesis of amino acids, steroid degradation, biosynthesis of secondary metabolites and biosynthesis of antibiotics were only significantly enriched in the probiotic supplemented group at production phase of life (Additional file 13: Fig. S8).

\section{Flock performance affected by probiotic supplementation}

The flock performance raw data obtained from the farm manager were visualised in Excel. The data showed that the weekly feed conversion ratio (FCR) was higher for the probiotic supplemented compared with the control flock (Additional file 14: Fig. S9). The egg production measured as the rate of lay (\%) and egg weight (g) from week 18 until week 36 of flock age were not different between the control and probiotic supplemented flocks (Additional file 15: Fig. S10).

Average body weight (kg) measured on 100 birds at weekly intervals was not different between the control and probiotic supplemented flocks (Additional file 16: Fig. S11). Eggs collected at 24, 30 and 36 week of flock age were processed for egg quality parameters. The probiotic supplementation significantly $(P<0.05)$ improved egg internal quality but not shell quality (Additional file 17: Fig. S12 a - f). The overall quality of albumen height, Haugh Unit and yolk colour was 


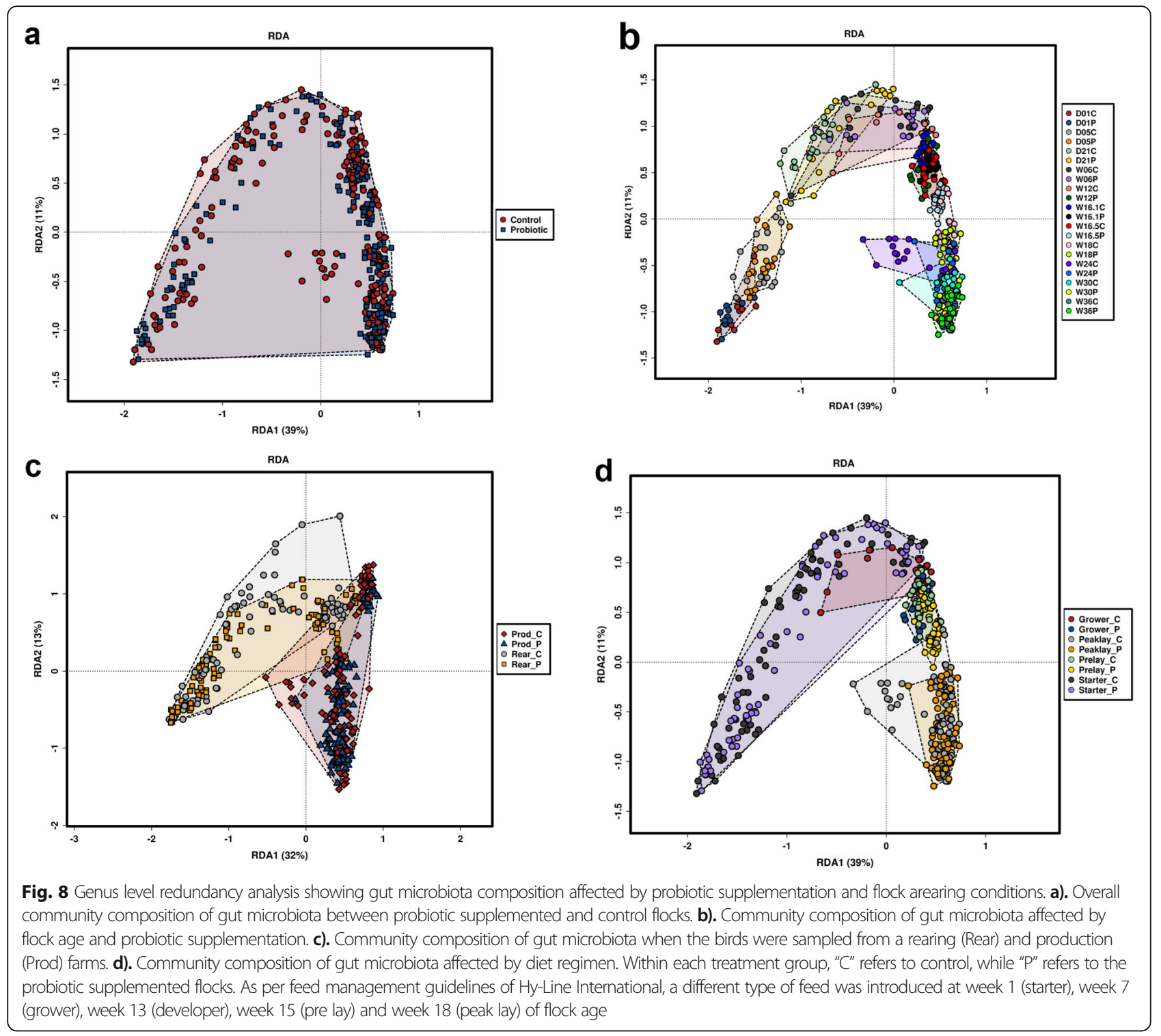

significantly higher in the probiotic supplemented flock compared to the control flock (Additional file 17: Fig. S12 d - f).

\section{Effect of Bacillus based probiotic on Salmonella in free-range layer production}

No Salmonella was isolated from the rearing and production sheds prior to placing the day-old chicks or point of lay pullets. Throughout the sampling period, Salmonella was isolated from one faecal sample and one environmental sample from the control shed at 18 and 36 weeks of flock age. At week 36, a shoe cover from the control shed was also positive for Salmonella spp. During the sampling period (day 1 to week 36 of flock age), no Salmonella was isolated from the probiotic supplemented shed. Measured through the mMPN method, a load of Salmonella in the faecal swab, environmental swab and shoe cover was $7.357,11.170$ and 15.06 per $\mathrm{mL}$ of BPW, respectively.

\section{Salmonella serotype confirmation through PCR}

The PCR and agarose gel electrophoresis results confirmed that one faecal sample collected from the control shed at 18 weeks of flock age was Salmonella Typhimurium, while one each of the environmental and shoe cover samples collected at 36 weeks of flock age from the control shed was non Typhimurium serotypes (Additional file 18: Fig. S13). 


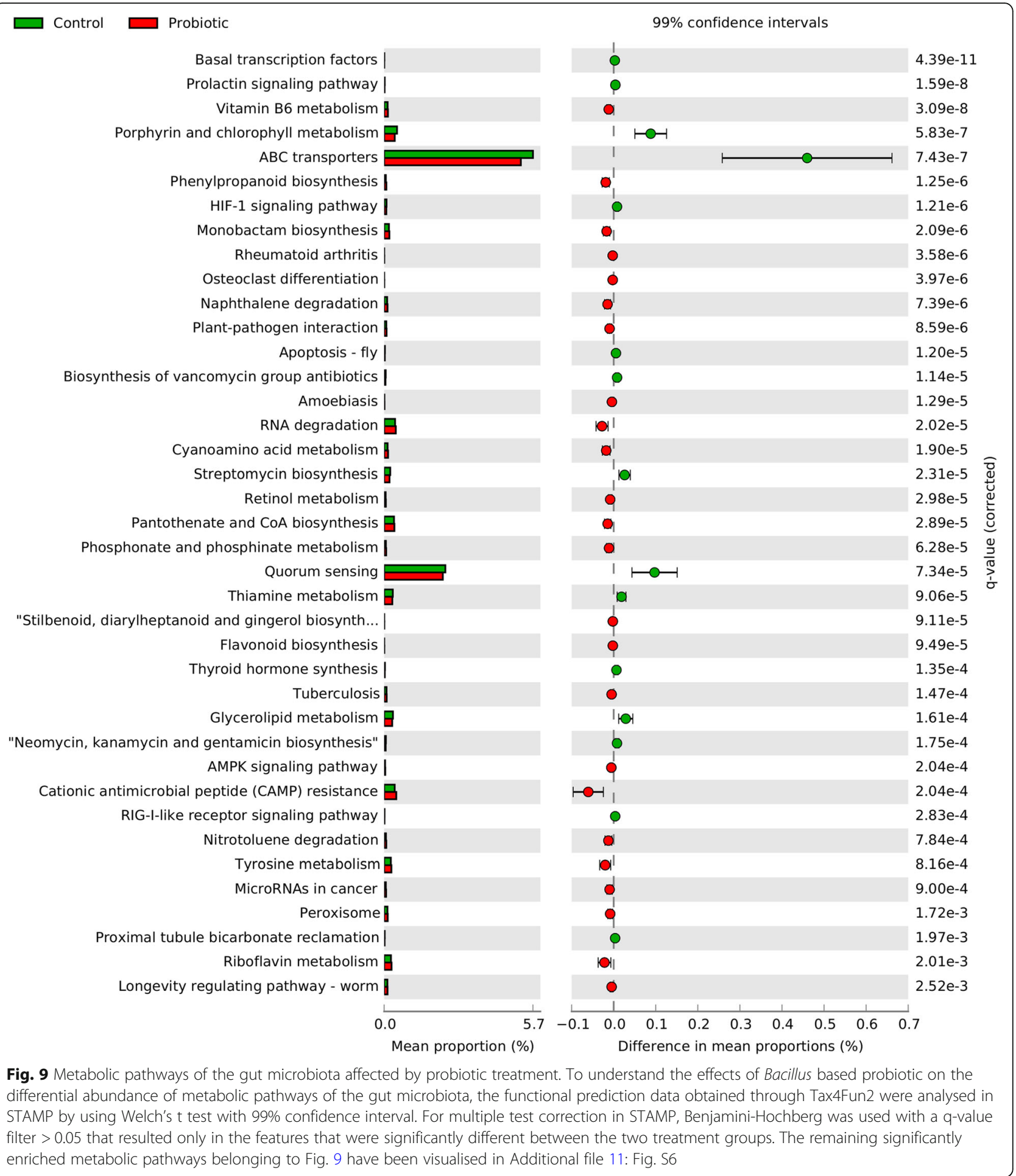

\section{Discussion}

In the laying hen industry, probiotics are mainly used as feed supplements for improving flock's performance; therefore, there is a need to understand the effects of direct-fed microbials on the composition and development of gut microbiota both in the rearing and production phases of cage layers reared in the field conditions. Also, not many longitudinal field trials have investigated the effects of probiotics supplementations on the gut microbiota of free range hens. The composition and the development of gut microbiota rapidly change in the first few weeks of chicken age [17, 41, 42] and 
introducing the right type of probiotic candidate at hatch may influence it positively, which could reflect in better performance of the flock. Considering the continuous supplementation of Bacillus based probiotic for reduction of pathogen load in the faeces is important for flock productivity and food safety [6], in the current study, we investigated the role of Bacillus based probiotic in free range production system. The data showed that the probiotic was effective in the abundance of microbial genera of gut microbiota both in the rearing and production phases of layers. However, the gut microbiota composition also changed as the flocks get advanced in age.

At the phylum level, the composition of gut microbiota dominated by Bacteroidetes, Firmicutes and Proteobacteria show the important role of these bacteria in gut health. These data also show Proteobacteria is the third dominant phylum in the gut of chickens. In a previous study, Bacteroidetes, Firmicutes and Proteobacteria have been shown as the main dominant phyla in the caecal content of laying chickens [41]. A 3.07\% increase in the overall population of Bacteroidetes in the probiotic supplemented flock shows its useful effects on gut health. Bacteroidetes are comprised of many bacteria that have the ability to digest complex substrates, such as xylan [43] and cellulose [44]. Microbial communities in gastrointestinal Bacteroidetes primarily produce propionate [45] and succinate [46] that are involved in intestinal gluconeogenesis. Proteobacteria was the third most abundant component of the gut microbiota with an overall $3.11 \%$ increase in the probiotic supplemented flock. This increase could be partly attributed to Parasutterella, which showed higher abundance in the probiotic flock. Proteobacteria also contain opportunistic pathogens, such as Campylobacter, Escherichia, Shigella, Salmonella and Helicobacter. In the current study, the abundance levels of these bacteria were more affected by flock age than the probiotic except Helicobacter that was significantly higher in the probiotic supplemented birds. The role of Helicobacter as a disease causing agent in chickens and its transmission through chicken meat and eggs needs to be investigated. A 5.95\% decrease in the overall population of Firmicutes shows that the probiotic supplementation reduced the abundance of certain bacteria including Peptostreptococcus $(0.323 \%$ in control versus $0.069 \%$ in probiotic). The increased abundance of Bacteroidetes in the gut at the production stage compared with the rearing both in the control and probiotic flocks shows age driven effects. Interestingly, the probiotic effects on the abundance level of Bacteroidetes were higher in the production compared with the rearing stage. The decreased abundance of Firmicutes after week 16 of flock age might explain the increase in Bacteroidetes that contains both succinate and propionate producing microbial communities.
The non-significant difference in alpha diversity (measured by Shannon index) between the probiotic supplemented and control flocks showed that microbial communities were more evenly distributed in the rearing phase. Probiotic supplementation did not significantly affect the alpha diversity (measured by Shannon index) of most of the faecal samples collected at different timepoints. An overall significantly higher alpha diversity of microbial communities in production compared with the rearing phase highlights the role of rearing conditions. A steady increase in the alpha diversity index of the probiotic supplemented and control flocks with age shows that age had profound effect on community structure within chickens. However, beta diversity showed a reduction trend with flock age, suggesting that as the chickens increased in age, the variation in the gut microbial communities between birds reduced. Beta diversity was also lower for the probiotic supplemented flock (Anosim Bray-Curtis $P=0.001$ ). Interestingly, assessing the effects of transportation of birds from rearing to production sheds on the beta diversity of gut microbiota showed that variation in the community structure increased in the probiotic supplemented flock posttransportation.

The intestinal microbiota is involved in the fermentation process of complex carbohydrates, such as indigestible dietary fibers and sugars to generate SCFAs that help in maintaining a healthy gut environment [13]. In the current study, overall, probiotic supplementation increased the abundance levels of many microbial communities in the gut. The increased abundance levels of Parasutterella, Paraprevotella, Megasphaera, Elusimicrobium, Succinatimonas, Desulfovibrionaceae_unclassified and Muribaculaceae_ge show that chickens performance was enhanced as these bacteria are involved in a range of useful functions in the gut of the host. Characterised in the gut of mice, Parasutterella plays a role in the maintenance of bile acid and cholesterol metabolism [47]. In our previous study, probiotic supplementation increased the abundance level of Parasutterella in the gut of Salmonella Typhimurium challenged laying chickens [6]. Parasutterella has shown to increase in abundance in the gut of pigs that received a prebiotic (waxy corn starch) supplemented diet [48], and in the gut of SPF chickens that received Lactobacillus casei supplemented diet [49]. In laying chickens gut, Paraprevotella is involved in polysaccharide degradation, and propionate and butyrate production through the expression of xylose isomerase, cobalamin-binding methylmalonylCoA mutase and/or methylmalonyl-CoA epimerase and acetyl-CoA acetyltransferase, respectively [50]. Megasphaera preferably produces butyrate through the production of acetyl-CoA acetyltransferase [50]. Elusimicrobium is a member of the phylum Elusimicrobia, 
which was the least abundant both in the control and probiotic supplemented flocks. Elusimicrobium minutum is the only identified specie in the Elusimicrobia and is an obligatory anaerobic ultramicrobacterium that ferments glucose [51]. Succinatimonas is a strict anaerobe that produces succinate and acetate from carbohydrates [52]. Members of Desulfovibrionaceae oxidise organic substrates incompletely to acetate [53]. In 60-week old Hy-Line Brown layers, the probiotic B. licheniformis alleviated the adverse influence of heat on egg production and gut health [54]. Bacillus subtilis strain DSM29784 has been shown to selectively increase the abundance of gut microbial genera, such as Bifidobacterium, Lactobacillus and Alistipes in different growing stages of layers [55]. The current study shows that the increased abundance of many of these microbial genera in the gut shows the useful aspects of the Bacillus based probiotic supplementation in the diet of layers.

In the functional prediction data, the significant enrichment of metabolic pathways, such as vitamin B6 metabolism, cyanoamino acid metabolism, retinol metabolism, lipopolysaccharide metabolism and biosynthesis of secondary metabolites show that probiotic supplementation improved gut functions. Vitamin B6 contributes to intestinal immune regulation through the metabolism of lipid mediator sphingosine 1-phosphate. Microbial vitamin B6 is synthesized as pyridoxal phosphate from d-ribulose 5-phosphate and glyceraldehyde3-phosphate or 4-phosphohydroxy-L-threonine and deoxyxylulose 5-phosphate [56]. Several natural isolates and engineered bacteria including Bacillus subtilis, produce vitamin B6 [57].

Different strains of probiotics vary in their mechanistic actions; therefore, we have only compared the findings of the current study with studies that used Bacillus based probiotics in layers. In laying hens, diet supplemented with Bacillus based probiotics has shown to improve egg production and overall egg quality. For example, supplementation of Bacillus subtilis but not Bacillus licheniformis in layer diet resulted in improved egg internal quality and egg production [58]. Feeding Bacillus licheniformis to a 28-week old layers flock for up to 8 weeks resulted in increased egg production, shell thickness and better intestinal epithelial cell morphology [59]. In 24-wk-old Lohmann Pink layers, Bacillus subtilis supplementation for up to 8 weeks resulted in improved FCR and reduced serum cholesterol level [60]. In the current study, the significantly higher albumen height, Haugh Unit and yolk colour show the positive effects of the Bacillus based probiotic on egg internal quality parameters; however, it is worth mentioning that the egg quality was measured only at week 24,30 and 36 weeks of flock age. The positive effects of probiotics could be due to improved nutrient absorption in the gut $[61,62]$ or through the production of metabolites, enzymes or vitamins $[63,64]$. Egg quality deteriorates with flock age; therefore, future research can focus on the use of probiotics for improving gut health and birds performance from hatch to the end of the production cycle.

No Salmonella detected prior to the placement of dayold chicks on rearing and 16-week pullets on production farms demonstrates that the decontamination procedures performed were appropriate. Effective cleaning and disinfection of poultry sheds reduce the levels of Salmonella contamination; however, the recovery of Salmonella spp. from surfaces such as dropping boards and floors in cleaned and disinfected sheds is variable [65]. In a previous study, different Salmonella serotypes such as, Salmonella Mbandaka and Salmonella Typhimurium were isolated from a free-range layer production system [65]. In the current study, no Salmonella isolation from the probiotic supplemented shed could be attributed to the beneficial effects of probiotics; however, a conclusive statement could not be made as the flock was not followed beyond 36 week of age. Moreover, the number of Salmonella positive samples were low $(n=3)$; therefore, it is hard to estimate the true effects of the Bacillus based probiotic on the flock Salmonella spp. status. In a pen trial, feeding of Bacillus based probiotic reduced the overall load of Salmonella in faeces but not all of the birds turned negative for Salmonella in the 12 week period of sampling [6].

\section{Conclusions}

In this field study, the most abundant bacterial phyla were Bacteroidetes, Firmicutes, and Proteobacteria in the gut microbiota. The probiotic supplementation from day-old significantly decreased overall abundance levels (\%) of Firmicutes and Spirochaetes and increased Elusimicrobia. At the genus level, the higher abundance of Elusimicrobium, Megasphaera, Parasutterella, Desulfovibrionaceae_unclassified, Paraprevotella, Succinatimonas, bacteria_unclassified, and Muribaculaceae_ge in the probiotic supplemented flock shows its positive effects on gut health. The functional enrichment of metabolic pathways including cationic antimicrobial peptide (CAMP) resistance, vitamin B6 metabolism, AMPK signaling pathway, monobactam biosynthesis, RNA degradation, and tyrosine metabolism highlights the positive effects of the Bacillus based probiotic on the gut. The improvement in egg internal quality parameters suggests that the Bacillus based probiotic could be used continuously in a cage-free poultry production system. Exploring suitable alternative multidisciplinary programs that encompass efficient management as well as optimize nutrition and disease management is of great interest to the poultry industry. Findings from this study can play a significant role in creating an antibiotic-free production environment. 


\section{Abbreviations}

BPW: Buffered peptone water; CSS: Cumulative-sum scaling; KEGG: Kyoto Encyclopedia of genes and genomes; NCBI: National Centre for Biotechnology Information; OTU: Operational taxonomic unit; RVS: Rappaport-Vassiliadis soya peptone; SCFAs: Short chain fatty acids; SRA: Sequence read archive; STAMP: Statistical analysis of taxonomic and functional profile

\section{Supplementary Information}

The online version contains supplementary material available at https://doi. org/10.1186/s42523-021-00112-5.

Additional file 1. Layer chicken diet composition, isolation of

Salmonella from swabs, faecal DNA extraction and traditional PCR

Additional file 2. Quality of reads generated in the study.

Additional file $\mathbf{3}$ Fig. S1. Rarefaction curve analysis of OTUs depicting the quality of the 165 rRNA reads generated from DNA obtained from laying chickens faeces. a) Rarefaction curve analysis of the reads based on the probiotic supplemented and control cohorts. b) Rarefaction curve analysis of the reads based on samples collected at Day (D) and Week (W) post-hatch from the probiotic supplemented (P) and control (C) groups. The flatten curves towards right show that the underlying microbial communities were well covered by the sequenced data.

Additional file 4. OTU Table containing details of taxa of faecal samples collected from the probiotic supplemented and control groups at different sampling time-points. (SUMMARY 1369 kb)

Additional file 5. Metadata file required for the OTU Table for further analysis and data visualisation. (CSV $52 \mathrm{~kb}$ )

Additional file 6 Fig. S2. Overall gut microbiota composition at phylum level in both the probiotic supplemented and control flocks pooled together. Microbial abundance (\%) in the probiotic supplemented group. For percent calculation of microbial abundance at phylum level, total sum scaling normalised but untransformed data obtained from Calypso software were visualised in Excel 2016 and panel graphs were prepared in Graphpad prism v. 8.0.0.

Additional file $\mathbf{7}$ Fig. S3. Abundance of gut microbiota at genus level affected by probiotic supplementation at multiple sampling periods. a). Bacteroides. b). Rikenellaceae_RC9 gut group. c). Lactobacillus. d). Alistipes. In each panel of the Supplementary Fig. S3, the letters " $D$ " and " $W$ " refer to day and week post-hatch, while the letters " $\mathrm{C}$ " and " $\mathrm{P}$ " refer to control and probiotic supplemented groups, respectively.

Additional file 8 Fig. S4. Abundance of gut microbiota at genus level affected by probiotic supplementation at multiple sampling periods. a). EscherichiaShigella. b). Enterococcus. c). Clostridium sensu stricto. d). Enterococcaceae_unclassified. In each panel of the Supplementary Fig. S4, the letters " $\mathrm{D}$ " and " $\mathrm{W}$ " refer to day and week post-hatch, while the letters " $C$ " and " $P$ " refer to control and probiotic supplemented groups, respectively.

Additional file 9 Fig. S5. Alpha diversity of gut microbiota of laying chickens affected by probiotic supplementation, rearing and laying conditions. a). Alpha diversity (Shannon index) of gut microbiota affected by feeding regimen. b). Richness of gut microbiota affected by feeding regimen. c). Evenness of gut microbiota affected by feeding regimen. d). Alpha diversity (Shannon index) of gut microbiota affected by transport stress. e). Richness of gut microbiota affected by transport stress. f). Evenness of gut microbiota affected by transport stress. Within each treatment group, " $\mathrm{C}$ " refers to control, while " $\mathrm{P}$ " refers to the probiotic supplemented cohort. Alpha diversity was measured at genus level by Shannon index, Richness and Evenness in Calypso software. Within each treatment group at specific sampling period, asterisk ${ }^{*}$ ) shows a significant difference at $P<0.01$, while asterisks $\left(^{* *}\right)$ show $P<0.001$.

Additional file 10. Details of metabolic pathways included in the functional prediction analysis. (XLS $3419 \mathrm{~kb}$ )

Additional file 11 Fig. S6. Metabolic pathways of the gut microbiota affected by probiotic supplementation. To understand the effect of Bacillus based probiotic on the differential abundance of metabolic pathways of the gut microbiota, the functional prediction data obtained through Tax4Fun2 were analysed in STAMP by using Welch's t test with 99\% confidence interval. For multiple test correction in STAMP,

Benjamini-Hochberg was used with q value filter $>0.05$ that resulted only in the features that were significantly different between the two treatment groups.

Additional file 12 Fig. S7. Metabolic pathways of the gut microbiota affected by probiotic supplementation in the rearing phase of laying flock. To understand the effect of Bacillus based probiotic on the differential abundance of metabolic pathways of the gut microbiota, the functional prediction data obtained through Tax4Fun2 were analysed in STAMP by using Welch's t test with $99 \%$ confidence interval. For multiple test correction in STAMP, Benjamini-Hochberg was used with q value filter $>0.05$ that resulted only in the features that were significantly different between the two treatment groups. In the figure, titles Rear_C and Rear_P refer to faecal samples collected from the control and probiotic supplemented cohorts at rearing phase of flock age, respectively.

Additional file 13 Fig. S8. Metabolic pathways of the gut microbiota affected by probiotic supplementation in the rearing phase of laying flock. To understand the effect of Bacillus based probiotic on the differential abundance of metabolic pathways of the gut microbiota, the functional prediction data obtained through Tax4Fun2 were analysed in STAMP by using Welch's t test with $99 \%$ confidence interval. For multiple test correction in STAMP, Benjamini-Hochberg was used with q value filter $>0.05$ that resulted only in the features that were significantly different between the two treatment groups. In the figure, titles Prod_C and Prod $\mathrm{P}$ refer to faecal samples collected from the control and probiotic supplemented cohorts at production phase of flock age, respectively.

Additional file $\mathbf{1 4}$ Fig. S9. Feed conversion ratio of the probiotic supplemented and control flocks. Weekly (W) feed conversion ratio was calculated from feed intake and flock body weight.

Additional file 15 Fig. S10. Lay rate and egg weight of probiotic supplemented and control flocks.

Additional file 16 Fig. S11. Body weight of probiotic supplemented and control flocks. Body weight of 100 randomly selected chickens was taken weekly (W) from each of the probiotic supplemented and control flocks.

Additional file 17 Fig. S12. Egg quality parameters of eggs collected from probiotic supplemented and control cohorts. a) Egg weight (g). b) Shell weight $(\mathrm{g})$. c) Shell thickness $(\mathrm{mm})$. d) Albumen height $(\mathrm{mm})$. e) Haugh Unit. f) Yolk colour. Freshly laid eggs were collected at week 24, 30 and 36 week of flock age and immediately analysed for egg shell and internal quality parameters.

Additional file $18 \mathrm{Fig}$. S13. PCR products of Salmonella positive samples visualised on $\mathbf{2} \%$ agarose gel. L) Ladder; $\mathbf{1})$ Salmonella Typhimurium isolated from faecal swab at week 18 of flock age from the control shed. 2) Salmonella spp. isolated from environmental swab isolated at week 36 of flock age from the control shed; 3) Salmonella spp. isolated from environmental swab isolated at week 36 of flock age from the control shed. 4) Salmonella Typhimurium as positive control. For Salmonella spp. typing primer pair (605 bp) amplifying the fragment of invA gene were used, while for Salmonella Typhimurium confirmation, primer pair (303 bp) amplifying the fragment of TSR3 gene were used.

\section{Acknowledgements}

We highly acknowledge Mrs. Sonali Deshmukh help in DNA extraction from chicken faecal samples. Help from Mr. Jiawei Zhang in samples collection in highly acknowledged. We also acknowledge the cooperation of an independent layer poultry producer located in South Australia by allowing us to perform this trial in free-range production system and providing us the performance data of the two flocks included in this study. Help from Bernd Wemheuer (Genomic and Applied Microbiology and Göttingen Genomics Laboratory, Institute of Microbiology and Genetics, University of Göttingen, Germany) in functional prediction data analysis is highly acknowledged.

\section{Authors' contributions}

KKC and SK contributed to the study design, samples collection, samples processing and critical revision of the manuscript. SK processed the samples, 
analysed the data and drafted the manuscript, while KKC critically edited it. Both the authors approved the manuscript for publication.

\section{Funding}

This research was financially supported by the Australian Eggs under the grant number 1FS802UA.

\section{Availability of data and materials}

The $16 \mathrm{~S}$ rRNA sequence data are available from the NCBI SRA under the BioProject accession number PRJNA647475. The sample metadata and OTU Tables are provided as Supplementary data files 1-2 for reproducibility of this study.

\section{Declarations}

\section{Ethics approval and consent to participate}

The experimental setup was approved by the Animal Ethics Approval Committee of The University of Adelaide, under Approval Number S-2019109. The protocol was carried out in accordance with the guidelines specified in Australian Code for the Care and Use of Animals for Scientific Purposes 8th edition 2013.

\section{Consent for publication}

Not applicable.

\section{Competing interests}

The authors declare that they have no competing no financial competing interests.

Received: 18 February 2021 Accepted: 7 July 2021

Published online: 27 July 2021

\section{References}

1. Yan W, Sun C, Yuan J, Yang N. Gut metagenomic analysis reveals prominent roles of Lactobacillus and cecal microbiota in chicken feed efficiency. Sci Rep. 2017;7(1):1-11.

2. Martinez-Guryn K, Hubert N, Frazier K, Urlass S, Musch MW, Ojeda P, et al. Small intestine microbiota regulate host digestive and absorptive adaptive responses to dietary lipids. Cell Host Microbe. 2018;23(4):458-469. e455.

3. Wen C, Yan W, Sun C, Ji C, Zhou Q, Zhang D, et al. The gut microbiota is largely independent of host genetics in regulating fat deposition in chickens. ISME J. 2019; 13(6):1422-36. https:/doi.org/10.1038/s41396-019-0367-2.

4. Donohoe DR, Garge N, Zhang X, Sun W, O'Connell TM, Bunger MK, et al. The microbiome and butyrate regulate energy metabolism and autophagy in the mammalian colon. Cell Metab. 2011;13(5):517-26. https://doi.org/10.1 016/j.cmet.2011.02.018.

5. Becattini S, Littmann ER, Carter RA, Kim SG, Morjaria SM, Ling L, et al. Commensal microbes provide first line defense against Listeria monocytogenes infection. J Exp Med. 2017;214(7):1973-89. https://doi.org/1 0.1084 /jem.20170495.

6. Khan S, Chousalkar KK: Salmonella typhimurium infection disrupts but continuous feeding of Bacillus based probiotic restores gut microbiota in infected hens. J Anim Sci Biotechnol 2020, 11(1):1-16, 29, DOl: https://doi. org/10.1186/s40104-020-0433-7.

7. Kampmann C, Dicksved J, Engstrand L, Rautelin H. Composition of human faecal microbiota in resistance to Campylobacter infection. Clin Microbiol Infect. 2016;22(1):61. e61-8.

8. Rakoff-Nahoum S, Paglino J, Eslami-Varzaneh F, Edberg S, Medzhitov R. Recognition of commensal microflora by toll-like receptors is required for intestinal homeostasis. Cell. 2004;118(2):229-41. https://doi.org/10.1016/j. cell.2004.07.002.

9. Bäckhed F, Ding H, Wang T, Hooper LV, Koh GY, Nagy A, et al. The gut microbiota as an environmental factor that regulates fat storage. Proc Natl Acad Sci U S A. 2004;101(44):15718-23. https://doi.org/10.1073/pnas.04 07076101.

10. Gill SR, Pop M, DeBoy RT, Eckburg PB, Turnbaugh PJ, Samuel BS, et al. Metagenomic analysis of the human distal gut microbiome. Science. 2006; 312(5778):1355-9. https://doi.org/10.1126/science.1124234.

11. Jacobson A, Lam L, Rajendram M, Tamburini F, Honeycutt J, Pham T, et al. A gut commensal-produced metabolite mediates colonization resistance to Salmonella infection. Cell Host Microbe. 2018;24(2):296-307. e297.
12. Mazmanian SK, Liu CH, Tzianabos AO, Kasper DL. An immunomodulatory molecule of symbiotic bacteria directs maturation of the host immune system. Cell. 2005;122(1):107-18. https://doi.org/10.1016/j.cell.2005.05.007.

13. Khan S, Moore RJ, Stanley D, Chousalkar KK. The gut microbiota of laying hens and its manipulation with prebiotics and probiotics to enhance gut health and food safety. Appl Environ Microbiol. 2020;86(13):e00600-20.

14. Russell WR, Duncan SH, Scobbie L, Duncan G, Cantlay L, Calder AG, et al. Major phenylpropanoid-derived metabolites in the human gut can arise from microbial fermentation of protein. Mol Nutr Food Res. 2013;57(3):52335. https://doi.org/10.1002/mnfr.201200594.

15. Elokil AA, Magdy M, Melak S, Ishfaq H, Bhuiyan A, Cui L, et al. Faecal microbiome sequences in relation to the egg-laying performance of hens using amplicon-based metagenomic association analysis. Animal. 2020;14(4): 706-15. https://doi.org/10.1017/S1751731119002428.

16. Ocejo M, Oporto B, Hurtado A. 165 rRNA amplicon sequencing characterization of caecal microbiome composition of broilers and freerange slow-growing chickens throughout their productive lifespan. Sci Rep. 2019;9(1):1-14.

17. Cui Y, Wang Q, Liu S, Sun R, Zhou Y, Li Y. Age-related variations in intestinal microflora of free-range and caged hens. Front Microbiol. 2017;8:1310. https://doi.org/10.3389/fmicb.2017.01310.

18. Ballou AL, Ali RA, Mendoza MA, Ellis J, Hassan HM, Croom W, et al. Development of the chick microbiome: how early exposure influences future microbial diversity. Front Vet Sci. 2016;3:2.

19. van der Eijk JAJ, de Vries H, Kjaer JB, Naguib M, Kemp B, Smidt H, et al. Differences in gut microbiota composition of laying hen lines divergently selected on feather pecking. Poult Sci. 2019;98(12):7009-21. https://doi.org/1 $0.3382 / \mathrm{ps} / \mathrm{pez} 336$

20. Shi D, Bai L, Qu Q, Zhou S, Yang M, Guo S, et al. Impact of gut microbiota structure in heat-stressed broilers. Poult Sci. 2019;98(6):2405-13. https://doi. org/10.3382/ps/pez026.

21. Joat NN, Khan S, Chousalkar K. Understanding the effects of intramuscular injection and feed withdrawal on Salmonella typhimurium shedding and gut microbiota in pullets. J Anim Sci Biotechnol. 2021;12(1):1-15.

22. Zhao L, Wang G, Siegel P, He C, Wang H, Zhao W, et al. Quantitative genetic background of the host influences gut microbiomes in chickens. Sci Rep. 2013;3(1):1163. https://doi.org/10.1038/srep01163.

23. Xu Y, Yang H, Zhang L, Su Y, Shi D, Xiao H, et al. High-throughput sequencing technology to reveal the composition and function of cecal microbiota in Dagu chicken. BMC Microbiol. 2016;16(1):1-9.

24. Wang J, Qm Z, WW N, Zhang X, Li Y, Al L, et al. Modulatory effect of Lactobacillus acidophilus KLDS 1.0738 on intestinal short-chain fatty acids metabolism and GPR41/43 expression in $\beta$-lactoglobulin-sensitized mice. Microbiol Immunol. 2019;63(8):303-15.

25. Castro MS, Molina MA, Azpiroz MB, Díaz AM, Ponzio R, Sparo MD, et al. Probiotic activity of Enterococcus faecalis CECT 7121: effects on mucosal immunity and intestinal epithelial cells. J Appl Microbiol. 2016;121(4):111729. https://doi.org/10.1111/jam.13226.

26. Lemme-Dumit JM, Cazorla SI, Perdigón GDV, Maldonado-Galdeano C. Probiotic Bacteria and their cell walls induce Th1-type immunity against Salmonella typhimurium challenge. Front Immunol. 2021;12:1770.

27. Lau LYJ, Chye FY. Antagonistic effects of Lactobacillus plantarum 0612 on the adhesion of selected foodborne enteropathogens in various colonic environments. Food Control. 2018;91:237-47.

28. Shi S, Qi Z, Gu B, Cheng B, Tu J, Song X, et al. Analysis of high-throughput sequencing for cecal microbiota diversity and function in hens under different rearing systems. 3. Biotech. 2019;9(12):1-11.

29. Chen S, Xiang H, Zhang H, Zhu X, Wang D, Wang J, et al. Rearing system causes changes of behavior, microbiome, and gene expression of chickens. Poult Sci. 2019;98(9):3365-76. https://doi.org/10.3382/ps/pez140.

30. Schloss PD, Westcott SL, Ryabin T, Hall JR, Hartmann M, Hollister EB, et al. Introducing mothur: open-source, platform-independent, community-supported software for describing and comparing microbial communities. Appl Environ Microbiol. 2009;75(23):7537-41. https://doi.org/10.1128/AEM.01541-09.

31. MiSeq SOP [https://www.mothur.org/wiki/MiSeq_SOP]. Accessed 10 Aug 2019.

32. Rognes T, Flouri T, Nichols B, Quince C, Mahé F. VSEARCH: a versatile open source tool for metagenomics. PeerJ. 2016;4:e2584. https://doi.org/10.7717/ peerj.2584.

33. Quast $C$, Pruesse E, Yilmaz $P$, Gerken J, Schweer $T$, Yarza $P$, et al. The SILVA ribosomal RNA gene database project: improved data processing and web- 
based tools. Nucleic Acids Res. 2012;41(D1):D590-6. https://doi.org/10.1093/ nar/gks1219.

34. Westcott SL, Schloss PD. OptiClust, an improved method for assigning amplicon-based sequence data to operational taxonomic units. mSphere. 2017;2(2):e00073-17.

35. Zakrzewski M, Proietti C, Ellis JJ, Hasan S, Brion M-J, Berger B, et al. Calypso: a user-friendly web-server for mining and visualizing microbiomeenvironment interactions. Bioinformatics. 2017;33(5):782-3. https://doi.org/1 0.1093/bioinformatics/btw725.

36. Paulson JN, Stine OC, Bravo HC, Pop M. Differential abundance analysis for microbial marker-gene surveys. Nat Methods. 2013;10(12):1200-2. https:// doi.org/10.1038/nmeth.2658.

37. Wemheuer F, Taylor JA, Daniel R, Johnston E, Meinicke P, Thomas T, et al. Tax4Fun2: prediction of habitat-specific functional profiles and functional redundancy based on 16S rRNA gene sequences. Environ Microbiome. 2020;15:1-12.

38. Parks DH, Tyson GW, Hugenholtz P, Beiko RG. STAMP: statistical analysis of taxonomic and functional profiles. Bioinformatics. 2014;30(21):3123-4. https://doi.org/10.1093/bioinformatics/btu494.

39. Samiullah S, Omar AS, Roberts J, Chousalkar K. Effect of production system and flock age on eggshell and egg internal quality measurements. Poult Sci. 2016;96(1):246-58. https://doi.org/10.3382/ps/pew289.

40. Haugh RR. The Haugh unit for measuring egg quality. United States Egg Poultry Magazine. 1937;43:522-55.

41. Videnska P, Sedlar K, Lukac M, Faldynova M, Gerzova L, Cejkova D, et al. Succession and replacement of bacterial populations in the caecum of egg laying hens over their whole life. PLoS One. 2014;9(12):e115142. https://doi. org/10.1371/journal.pone.0115142.

42. Joat N, Van TTH, Stanley D, Moore RJ, Chousalkar K. Temporal dynamics of gut microbiota in caged laying hens: a field observation from hatching to end of lay. Appl Microbiol Biotechnol. 2021;105(11):4719-30.

43. Dodd D, Mackie RI, Cann IK. Xylan degradation, a metabolic property shared by rumen and human colonic Bacteroidetes. Mol Microbiol. 2011;79(2):292304. https://doi.org/10.1111/j.1365-2958.2010.07473.x.

44. Vera-Ponce de León A, Jahnes BC, Duan J, Camuy-Vélez LA, Sabree ZL. Cultivable, host-specific Bacteroidetes symbionts exhibit diverse polysaccharolytic strategies. Appl Environ Microbiol. 2020;86(8):e0009100020.

45. De Vadder F, Kovatcheva-Datchary P, Goncalves D, Vinera J, Zitoun C, Duchampt A, et al. Microbiota-generated metabolites promote metabolic benefits via gut-brain neural circuits. Cell. 2014;156(1):84-96. https://doi. org/10.1016/j.cell.2013.12.016.

46. De Vadder F, Kovatcheva-Datchary P, Zitoun C, Duchampt A, Bäckhed F, Mithieux G. Microbiota-produced succinate improves glucose homeostasis via intestinal gluconeogenesis. Cell Metab. 2016;24(1):151-7. https://doi. org/10.1016/j.cmet.2016.06.013.

47. Ju T, Kong JY, Stothard P, Willing BP. Defining the role of Parasutterella, a previously uncharacterized member of the core gut microbiota. The ISME journal. 2019;13(6):1520-34. https://doi.org/10.1038/s41396-019-0364-5.

48. Metzler-Zebeli BU, Schmitz-Esser S, Mann E, Grüll D, Molnar T, Zebeli Q. Adaptation of the cecal bacterial microbiome of growing pigs in response to resistant starch type 4. Appl Environ Microbiol. 2015;81(24):8489-99. https://doi.org/10.1128/AEM.02756-15.

49. Tabashsum Z, Peng M, Alvarado-Martinez Z, Aditya A, Bhatti J, Romo PB, et al. Competitive reduction of poultry-borne enteric bacterial pathogens in chicken gut with bioactive Lactobacillus casei. Sci Rep. 2020;10(1):1-12.

50. Polansky O, Sekelova Z, Faldynova M, Sebkova A, Sisak F, Rychlik I. Important metabolic pathways and biological processes expressed by chicken cecal microbiota. Appl Environ Microbiol. 2016;82(5):1569-76. https://doi.org/1 0.1128/AEM.03473-15.

51. Brune A. The family Elusimicrobiaceae. The prokaryotes; 2014. p. 637-40.

52. Santos EO, Thompson F. The Family Succinivibrionaceae. In: Rosenberg E, EF DL, Lory S, Stackebrandt E, Thompson F, editors. The Prokaryotes: Gammaproteobacteria. Berlin: Springer Berlin Heidelberg; 2014. p. 639-48.

53. Kuever J. In: Rosenberg E, DeLong EF, Lory S, Stackebrandt E, Thompson F, editors. The family desulfovibrionaceae. The Prokaryotes. Berlin: Springer; 2014. p. 107-33.

54. Deng W, Dong XF, Tong JM, Zhang Q. The probiotic Bacillus licheniformis ameliorates heat stress-induced impairment of egg production, gut morphology, and intestinal mucosal immunity in laying hens. Poult Sci. 2012;91(3):575-82. https://doi.org/10.3382/ps.2010-01293.
55. Neijat M, Habtewold J, Shirley RB, Welsher A, Barton J, Thiery P, et al. Bacillus subtilis strain DSM 29784 modulates the cecal microbiome, concentration of short-chain fatty acids, and apparent retention of dietary components in shaver white chickens during grower, developer, and laying phases. Appl Environ Microbiol. 2019;85(14):e00402-19.

56. Magnúsdóttir S, Ravcheev D, de Crécy-Lagard V, Thiele I. Systematic genome assessment of B-vitamin biosynthesis suggests co-operation among gut microbes. Front Genet. 2015;6:148.

57. Rosenberg J, Ischebeck T, Commichau FM. Vitamin B6 metabolism in microbes and approaches for fermentative production. Biotechnol Adv. 2017;35(1):31-40. https://doi.org/10.1016/j.biotechadv.2016.11.004

58. Upadhaya SD, Rudeaux F, Kim IH. Efficacy of dietary Bacillus subtilis and Bacillus licheniformis supplementation continuously in pullet and lay period on egg production, excreta microflora, and egg quality of Hyline-Brown birds. Poult Sci. 2019;98(10):4722-8. https://doi.org/10.3382/ps/pez184.

59. Lei K, Li YL, Yu DY, Rajput IR, Li WF. Influence of dietary inclusion of Bacillus licheniformis on laying performance, egg quality, antioxidant enzyme activities, and intestinal barrier function of laying hens. Poult Sci. 2013;92(9): 2389-95. https://doi.org/10.3382/ps.2012-02686.

60. Zhang JL, Xie QM, Ji J, Yang WH, Wu YB, Li C, et al. Different combinations of probiotics improve the production performance, egg quality, and immune response of layer hens. Poult Sci. 2012;91(11):2755-60. https://doi. org/10.3382/ps.2012-02339.

61. Mikulski D, Jankowski J, Naczmanski J, Mikulska M, Demey V. Effects of dietary probiotic (Pediococcus acidilactici) supplementation on performance, nutrient digestibility, egg traits, egg yolk cholesterol, and fatty acid profile in laying hens. Poult Sci. 2012;91(10):2691-700. https://doi.org/10.3382/ps.201202370.

62. Panda AK, Rama Rao SS, Raju MVLN, Sharma SS. Effect of probiotic (Lactobacillus sporogenes) feeding on egg production and quality, yolk cholesterol and humoral immune response of white Leghorn layer breeders. J Sci Food Agric. 2008;88(1):43-7. https://doi.org/10.1002/jsfa.2921.

63. Crittenden RG, Martinez NR, Playne MJ. Synthesis and utilisation of folate by yoghurt starter cultures and probiotic bacteria. Int J Food Microbiol. 2003; 80(3):217-22. https://doi.org/10.1016/S0168-1605(02)00170-8.

64. Hill MJ. Intestinal flora and endogenous vitamin synthesis. Eur I Cancer Prev. 1997:6(2):S43-5. https://doi.org/10.1097/00008469-199703001-00009.

65. Carrique-Mas JJ, Marin C, Breslin M, McLaren I, Davies R. A comparison of the efficacy of cleaning and disinfection methods in eliminating Salmonella spp. from commercial egg laying houses. Avian Pathol. 2009;38(5):419-24. https://doi.org/10.1080/03079450903193768.

\section{Publisher's Note}

Springer Nature remains neutral with regard to jurisdictional claims in published maps and institutional affiliations.

Ready to submit your research? Choose BMC and benefit from:

- fast, convenient online submission

- thorough peer review by experienced researchers in your field

- rapid publication on acceptance

- support for research data, including large and complex data types

- gold Open Access which fosters wider collaboration and increased citations

- maximum visibility for your research: over $100 \mathrm{M}$ website views per year

At BMC, research is always in progress.

Learn more biomedcentral.com/submissions 